\title{
Jejunojejunal Lipoma Causing Intussusception
}

\author{
G. Charalambous ${ }^{a, b} \quad$ V. Katergiannakis ${ }^{a} \quad$ A. Manouras ${ }^{a}$ \\ ${ }^{a}$ First Propaedeutic Surgical Department, University of Athens, Athens, Greece; \\ ${ }^{b}$ School of Health Sciences, Frederick University, Pontoirakleias, Cyprus
}

\section{Key Words}

Lipoma $\cdot$ Jejunojejunal $\cdot$ Intussusception

\begin{abstract}
Jejunojejunal intussusception and jejunojejunal lipomas are both very rare clinical entities. The present case report describes this event in an adult patient with severe episodes of hematochezia and colicky upper abdominal pain. The diagnosis was not made preoperatively, as none of the endoscopic findings were pathognomonic and, furthermore, CT scans could not rule out malignancy. Exploratory laparotomy was mandatory and at surgery a jejunojejunal intussusception secondary to a lipoma was found. Segmental resection and primary reanastomosis were performed in the presence of evidence of bowel ischemia.
\end{abstract}

\section{Introduction}

Adult intussusception accounts for $5 \%$ of all intussusceptions [1]. Unlike in children, a lead point is usually found and it is frequently caused by a malignant small bowel lesion acting as the apex of the intussusception. Primary small intestinal tumors are uncommon and account for about $1 \%$ of all gastrointestinal tumors. Primary lipomas of the small intestine are also rare entities, representing $2.6 \%$ of nonmalignant tumors of the intestinal tract [2]. Most of them remain asymptomatic throughout life and only one third of these patients have symptoms related to the size of the tumor, or it is encountered incidentally during investigation of the gastrointestinal tract for another reason [3]. The majority of lipomas in the small bowel are solitary and, in the gastrointestinal tract, they may protrude as an intraluminal submucosal mass, which may lead to severe symptoms [4]. Three growth patterns have been identified: intraluminal, infiltrative and serosal. Intraluminal lesions are most often associated with the development of secondary bowel obstruction and intussusception, while serosal lesions are linked to small bowel volvulus. It is a relatively infrequent and even rarer source of gastrointestinal bleeding. Ulceration or vascular compromise due to an 
intussusception can cause acute blood loss [5, 6]. Preoperative diagnosis is usually indeterminate, only based on clinical manifestations due to the overlap of symptoms and signs with other intestinal tumors. Benign tumors in the small intestine do not have a characteristic CT appearance and, in most instances, cannot be differentiated from malignant lesions, but lipomas can be definitively diagnosed by the recognition of fat attenuation within the mass [7]. In such cases an attempt at reduction with local excision must always be performed, but if this is unobtainable, resection is indicated $[5,8]$.

The present report describes an unusual case of adult intussusception caused by lipoma of the jejunum with nonspecific abdominal symptoms and reviews some aspects of diagnosis and treatment of intestinal intussusception.

\section{Case Report}

A 46-year-old man, obese (145 kg), was admitted to our department with a 3-month history of intermittent mild rectal bleeding, as he reported the passing of a few drops of fresh blood that turned the toilet water pink. During this period he had two episodes of hematochezia following a crampy abdominal pain located in the upper abdomen focusing in the periumbilical region. The first episode brought him to his doctor but both physical and endoscopic examination revealed no special pathology. The patient denied having distension, past history of peptic ulcer disease, alteration in bowel habits, melena or weight loss. A complete review of systems plus his medical and family history were unremarkable. In addition, further diagnostic procedures including barium enema, angiography and tumor markers were normal. Nevertheless, comparison with previous laboratory tests proved a decrease in hemoglobin values from 14 to $12 \mathrm{~g} / \mathrm{dl}$. Blood transfusion was not administrated and, as the bleeding had stopped, exploratory laparotomy was contraindicated. The patient was free of symptoms for 3 months when he experienced a similar, but more severe episode accompanied by symptoms of weakness, dizziness, near-fainting, swelling and hypotension. On his second admission hemoglobin was $8.8 \mathrm{~g} / \mathrm{dl}$ and the pain had become more constant, colicky in nature, frequently recurring and increased in terms of intensity and duration. The repetition of gastroscopy and colonoscopy revealed no special findings, but a new CT examination showed evidence of a sausage-like mass, which presented as an oval well-circumscribed intraluminal filling defect, and bowel wall thickening (fig. 1). Abdominal conventional radiographs showed dilation of the jejunum with multiple air levels, involving small bowel loops in the left upper quadrant of the abdomen, and enteroclysis with Gastrografin demonstrated a circular obstructing lesion of the jejunum with apple core appearance.

As the nature of this lesion was not clear and malignancy could not be excluded, an exploratory laparotomy was performed. Surgically, $30 \mathrm{~cm}$ beyond the ligament of Treitz, a $4 \mathrm{~cm}$ polypoid mass was found, acting as the lead point of intussusception. Enterotomy following manual reduction of the intussusception revealed a $4 \times 4 \mathrm{~cm}$ pedunculated cylindrical lesion that was excised with a portion of lateral jejunal wall. The presence of severe bowel ischemia and the absence of vital signs in a small portion of the intussusception indicated segmental resection and primary reanastomosis as the procedure of choice ( $\underline{\text { fig. } 2}$ ).

Final histologic examination confirmed our diagnosis with the emergence of tissue with mature lipocytes corresponding to submucosal lipoma of the jejunum. The postoperative period was uneventful, and the patient was discharged on the seventh postoperative day. At 3 years following surgery he is free of symptoms and no evidence of recurrence has ever been noticed.

\section{Discussion}

Approximately 1.5-6\% of all gastrointestinal neoplasms occur as primary tumors of the small bowel $[5,6]$. The frequency of benign and malignant tumors is reported 
to be equal in surgical series whereas benign neoplasms by far exceed malignant neoplasms in autopsy series. Lipomas are benign mesenchymal tumors that arise from the submucosa in $90 \%$ of cases, while the remainder are subserosal. In the gastrointestinal tract the colon and the small bowel are afflicted in 70 and $25 \%$ of cases respectively. They are found in the ileum, duodenum and jejunum in decreasing order of frequency and are multiple in $18 \%$ of patients $[7,8]$.

Most intestinal lipomas are asymptomatic and only 33\% of them cause symptoms, which are always related to the size of the tumor. Tumors less than $1 \mathrm{~cm}$ are asymptomatic, while lesions larger than $4 \mathrm{~cm}$ may result in intermittent crampy abdominal pain, melena or hematochezia from ulceration of the overlying mucosa and intussusception secondary to a pedunculated lipoma. It has been reported that in the small bowel, lipomas account for $44 \%$ of all instances of intussusception. To our best knowledge, only 6 cases of jejunojejunal intussusception have been described $[9,10]$.

Preoperative diagnosis of the lesion, although desirable in facilitating the planning of surgery, is not always feasible as in our case. Nevertheless, many investigators suggest that the CT findings in intussusception are usually pathognomonic even if demonstration of the lead point is rare. The CT features include: (1) A target-like mass in which the inner central area represents the invaginated intussusceptum that is surrounded by the thick-wall intussuscipiens. (2) Oral contrast material trapped between the opposing walls of the intussusceptum and intussuscipiens. (3) A soft tissue mass secondary to the intussusception, possibly with the accompanying lead point telescoping into the intussuscipiens. (4) Bowel wall thickening or intramural air may be seen in case of blood supply restriction. A lipoma can be diagnosed if a smooth mass of fat attenuation ( -50 to $100 \mathrm{HU}$ ) is identified within the lumen of intussuscipiens. In addition, upper gastrointestinal series can reveal the presence of an intraluminal mass with the classic 'coiled spring' appearance of barium between the intussuscipiens and the entering intussusceptum $[11,12]$.

Endoscopic examination is often performed early in the diagnosis of gastrointestinal bleeding. Although it has little to offer in case of location of the lesion in the small intestine, it is very useful when the tumor is located in the colon. When tumors lie in the submucosa they have the typical endoscopic appearance of a smooth, hemispherical polypoid lesion with a wide base. In some other cases they appear to be pedunculated with a short and thick stalk. Multible biopsy specimens should be taken from the same site to denude mucosa and thereby expose the underlying fat. This has been described as the 'naked sign' and it is pathognomonic of lipoma. Endoscopic removal of lipomas has been described with snare cautery, but this is a difficult procedure with a $43 \%$ perforation rate in one recent series and it is also inadvisable if the nature of the lesion is uncertain [13].

Spontaneous elimination of gastrointestinal lipomas has been described since 1757. In reviewing these cases, the probable origin cited was the colon and the small intestine in 72 and $24 \%$ of cases respectively, while in $4 \%$ of them the origin cited was unknown. Complications associated with lipoma expulsion are persistent bleeding, colon perforation and intussusception. Only one case of spontaneous small bowel lipoma expulsion complicated with intussusception has been described in the world literature $[14,15]$. 
The treatment of symptomatic small bowel lipomas is surgical. Local excision, where feasible, should be performed in uncomplicated cases. If the tumor has caused intussusception and the attempt at reduction with local excision fails, segmental resection and primary reanastomosis is the procedure of choice. Frozen section of the specimen is always advisable in order to exclude the possible malignant nature of the lesion.
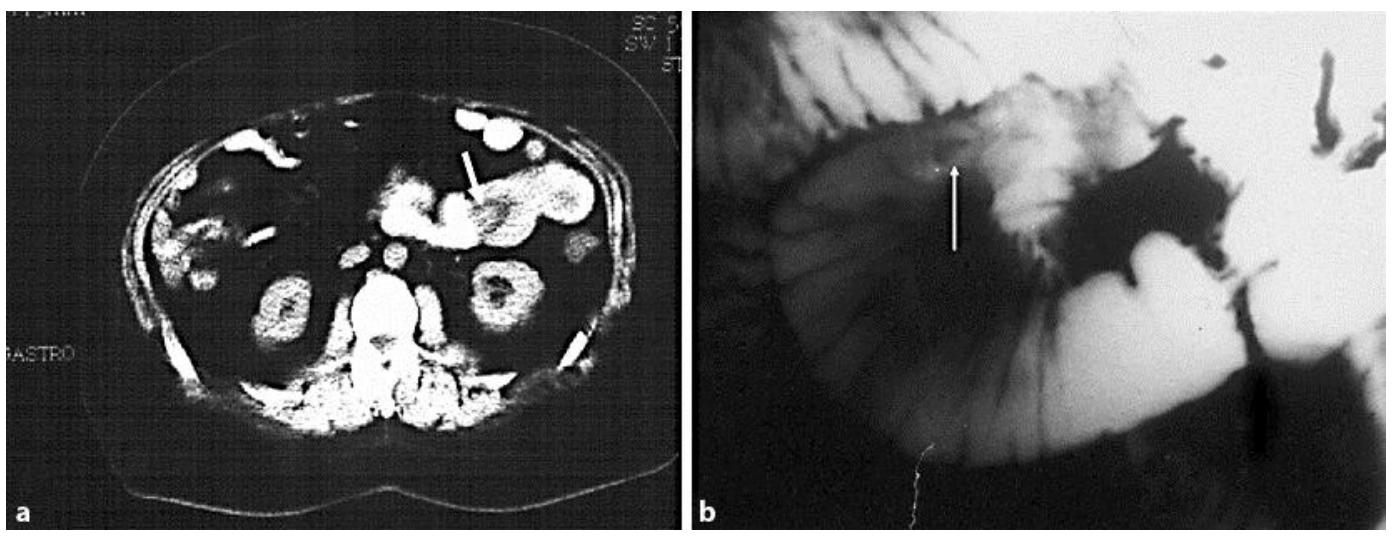

Fig. 1. a CT scan image of intussusception. b Enteroclysis shows an oval mass of the jejunum representing the lead point of the intussusception with dilatation of the thickened, edematous, adjacent bowel loops. The arrows show the apple core appearance.
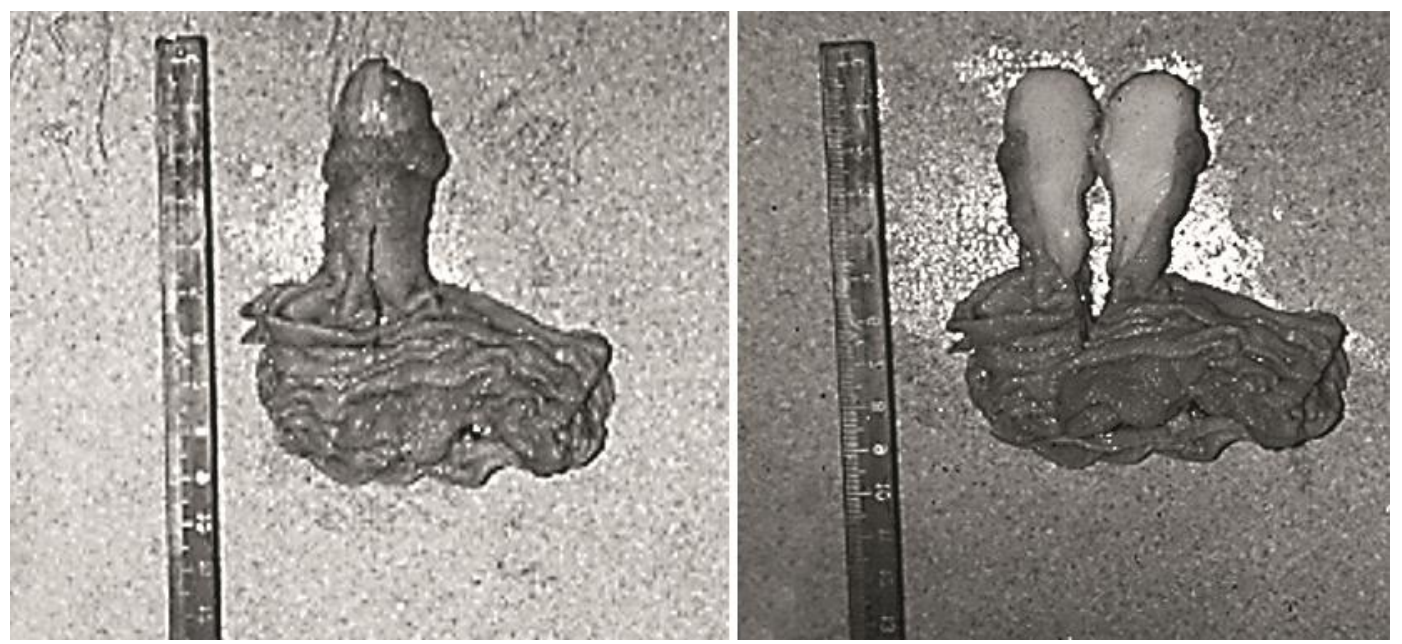

Fig. 2. Macroscopic appearance of the specimen after segmental resection. The gross pathologic specimen shows a jejunal loop containing a submucosal lipoma. Note that the mucosa was resected. The lesion was covered with submucosa. 


\section{References}

1 Weinberg T, Feldman M: Lipomas of the gastrointestinal tract. Am J Clin Path ol 1955;25:272-280.

-2 Ackerman NB, Chughtai SQ: Symptomatic lipomas of the gastrointestinal tract. Surg Gynecol Obstet 1975; 141:565-568.

3 Mayo CW, Pagtalunan RJG, Brown DJ: Lipoma of the alimentary tract. Surgery 1963;53:598-603.

4 Scottenfeld LE: Lipomas of the gastrointestinal tract. Surgery 1943;14:47-72.

5 Ketonen P, Virkkula L, Ketonen L, et al: Recurrent small bowel obstruction caused by a benign tumor. Acta Chir Scand 1978;144:261-262.

6 Gill SS, Heuman DM, Mihas AA: Small intestinal neoplasms. J Clin Gastroenterol 2001;33:267-282.

7 Lin F, Setya V, Signor W: Gastroduodenal intussusception secondary to a gastric lipoma: a case report and review of the literature. Am Surg 1992;58:772-774.

8 Notaro JR, Masser PA: Annular colon lipoma: a case report and review of the literature. Surgery 1991;110:570-572.

9 Weiss A, Mollura JL, Profy A, et al: Two cases of complicated intestinal lipomas. Am J Gastroenterol 1979;72:83-88.

10 Comfort MW: Submucous lipomata of the gastrointestinal tract: report of twenty-eight cases. Surg Gynecol Obstet 1931;52:108-118.

11 Urbano J, Serantes A, Hernandez L, et al: Lipoma-induced jejunojejunal intussusception: US and CT diagnosis. Abdom Imaging 1996;21:522-524.

12 Gayer G, Apter S, Hofman C, et al: Intussusception in adults: CT diagnosis. Clin Radiol 1998;53:53-57.

13 van Heel DA, Panos MZ: Colonoscopic appearances and diagnosis of intussusception due to large-bowel lipoma. Endoscopy 1999;31:508.

14 Gray Y, Jhung JW, Shapiro BA: Small intestinal intussusception secondary to a submucosal lipoma. Arch Pathol Lab Med 2002;126:231-232.

15 Freund HR: Lipoma of the small bowel. J Am Coll Surg 2000;191:465. 